\title{
T-Cell Immunoreceptor with Ig and ITIM Domains
}

National Cancer Institute

\section{Source}

National Cancer Institute. T-Cell Immunoreceptor with Ig and ITIM Domains. NCI

Thesaurus. Code C126125.

T-cell immunoreceptor with Ig and ITIM domains (244 aa, $26 \mathrm{kDa}$ ) is encoded by the human TIGIT gene. This protein is involved in immunomodulation and cell-cell adhesion. 